\title{
Prognostic significance of vascular endothelial growth factor and cyclooxygenase 2 expression in patients receiving preoperative chemoradiation for esophageal cancer
}

\author{
Matthew H. Kulke, $\mathrm{MD}^{\mathrm{a}}$ \\ Robert D. Odze, $M D^{\mathrm{b}}$ \\ James D. Mueller, $M D^{b}$ \\ Helen Wang, MD ${ }^{\mathrm{c}}$ \\ Mark Redston, $\mathrm{MD}^{\mathrm{b}}$ \\ Monica M. Bertagnolli, MD ${ }^{d}$
}

From the Department of Adult Oncology,
Dana-Farber Cancer Institute, Boston,
Mass; the Departments of Pathology ${ }^{\mathrm{b}}$ and
Surgery, Brigham and Women's Hospital,
Boston, Mass; and the Department of Pa-
thology, Beth Israel Deaconess Medical
Center, Boston, Mass.

Received for publication May 8, 2003; revisions requested Nov 28, 2003; accepted for publication Dec 30, 2003.

Address for reprints: Matthew H. Kulke, MD, Department of Adult Oncology, DanaFarber Cancer Institute, Boston, MA 02115 (E-mail: matthew_kulke@dfci.harvard.edu).

J Thorac Cardiovasc Surg 2004;127: 1579-86

$0022-5223 / \$ 30.00$

Copyright (๑) 2004 by The American Association for Thoracic Surgery

doi:10.1016/j.jtcvs.2003.12.034
Objective: Both vascular endothelial growth factor and cyclooxygenase 2 overexpression have been associated with poor prognosis in a variety of human malignancies. In this study we assessed the effect of preoperative chemotherapy and radiation on expression levels of vascular endothelial growth factor and cyclooxygenase 2 in patients with esophageal cancer and determined whether these markers were associated with treatment response and overall survival.

Methods: Expression levels of vascular endothelial growth factor and cyclooxygenase 2 were measured in a cohort of 46 patients with esophageal cancer receiving preoperative chemoradiation followed by surgical resection. Immunohistochemical stains were performed on both pretreatment biopsy specimens and posttreatment resection specimens for each patient. Differences in vascular endothelial growth factor and cyclooxygenase 2 expression before and after treatment were measured, and pretreatment expression levels were correlated with treatment response and overall survival.

Results: We found that preoperative chemotherapy and radiation induced expression of cyclooxygenase 2 in stromal cells and induced vascular endothelial growth factor expression in both tumor and stromal cells. Pretreatment vascular endothelial growth factor expression did not correlate with treatment response, and cyclooxygenase 2 expression correlated with treatment response only in the subset of patients with squamous cell carcinoma. Although patients whose tumors expressed high levels of vascular endothelial growth factor and cyclooxygenase 2 tended to have shorter overall survival times, this trend did not reach statistical significance.

Conclusions: Neither vascular endothelial growth factor nor cyclooxygenase 2 are strong predictors of treatment response and survival in patients undergoing preoperative chemoradiation for esophageal cancer. This lack of prognostic significance might be explained by changes in the expression levels of these markers during treatment.

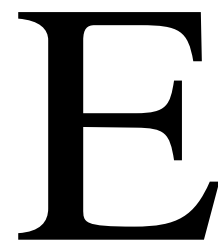

sophageal cancer is the cause of more than 12,000 deaths in the United States per year and is increasing in incidence. ${ }^{1}$ Unfortunately, only $10 \%$ of patients given a diagnosis of esophageal cancer are cured of their disease. Preoperative chemotherapy and external beam radiation is a common form of treatment for patients who have surgically resectable and therefore potentially curable esophageal cancer. With this approach, patients who achieve complete pathologic responses 
after preoperative therapy have a better prognosis than those who do not respond. ${ }^{2,3}$ This observation has led to the investigation of markers that might help predict response to preoperative chemotherapy and radiation and thus might allow selection of patients who could benefit from an aggressive treatment regimen.

Both tumor cells and surrounding stromal cells are known to secrete a variety of cytokines and growth factors, many of which have been shown to have long-term prognostic significance. One such cytokine is vascular endothelial growth factor (VEGF), which induces endothelial cell proliferation and vascular permeability and is associated with increased microvessel density. ${ }^{4,5}$ VEGF is overexpressed in several human solid tumor types, including $30 \%$ to $60 \%$ of esophageal cancers. ${ }^{6-9}$ In patients undergoing surgical resection for squamous cell carcinoma of the esophagus, multiple studies have demonstrated a correlation between high levels of VEGF expression, advanced stage, and poor overall survival. ${ }^{6-10}$

The value of VEGF expression as a predictor of treatment response in patients receiving preoperative chemotherapy and radiation therapy for esophageal cancer is less clear. Two studies have suggested that VEGF overexpression is associated with resistance to treatment. ${ }^{11,12}$ A third study, however, failed to confirm these findings. ${ }^{13}$ Furthermore, a study examining microvessel density in patients with esophageal cancer receiving preoperative chemoradiation showed no correlation with treatment response or survival. ${ }^{14}$ These studies suggest that VEGF is a less useful prognostic marker in patients undergoing preoperative therapy.

Cyclooxygenase 2 (COX-2) expression has also been evaluated, both as a potential predictor of response to chemotherapy and radiation and as a predictor of overall survival. COX-2 is one of several enzymes responsible for arachidonic acid metabolism and is induced by a number of growth factors and cytokines. ${ }^{15} \mathrm{COX}-2$ has been implicated in the promotion of angiogenesis, inhibition of cell-cycle control, and resistance to apoptosis. ${ }^{15,16} \mathrm{COX}-2$ overexpression has also been associated with resistance to preoperative systemic cisplatin-based chemotherapy and concurrent radiation therapy in patients with squamous cell carcinoma of the cervix and has been shown to predict shorter survival times in patients with cancers of the stomach, breast, bladder, cervix, ovary, and head and neck. ${ }^{17-23}$ In patients with esophageal adenocarcinoma undergoing surgical resection, COX-2 overexpression has been associated with a higher probability of metastasis and shorter overall survival times. ${ }^{24}$ The value of COX-2 as a prognostic factor in patients with esophageal cancer undergoing preoperative chemotherapy and radiation has not been studied.

Although both VEGF and COX-2 overexpression are clear adverse prognostic factors in other settings, the potential for preoperative therapy to induce changes in the ex- pression levels of VEGF and COX-2 might diminish their utility as prognostic factors in patients receiving such treatment. We therefore measured VEGF and COX-2 expression in esophageal cancer tissue and tumor-associated stroma from 46 patients, all of whom had received preoperative chemotherapy and radiation. We determined whether the pretreatment expression levels were associated with either treatment response or overall survival and assessed whether preoperative therapy induced changes in the expression levels of these markers.

\section{Materials and Methods \\ Patients}

The study group consisted of 46 patients with esophageal cancer who were identified through a review of medical records as having received concurrent neoadjuvant chemotherapy and radiation followed by esophageal resection at the Brigham and Women's Hospital between 1989 and 1995 and for whom both pretreatment biopsy specimens and posttreatment resection specimens could be evaluated for marker expression. The study was conducted with institutional review board approval. Follow-up survival and disease recurrence data were available for all patients and were obtained either through a review of patient charts or by contacting the patients directly.

\section{Specimen Collection}

All esophageal resection specimens were received in the surgical pathology laboratory in the fresh state and then fixed in $10 \%$ buffered formalin for subsequent tissue sectioning. The specimens were sectioned according to a standard protocol, which included a minimum of 3 sections through the deepest portion of the tumor, a minimum of 3 sections through the proximal and distal margins of resection, and a thorough lymph node sampling. For cases in which no gross tumor was identified (eg, patients with complete pathologic response after chemotherapy and radiation), the entire area of ulcerated or otherwise abnormal-appearing tissue was submitted for histologic evaluation.

\section{Tissue Analysis}

Five-micrometer-thick tissue sections of tumor were cut from formalin-fixed, paraffin-embedded tissue blocks and used for further immunohistochemical analysis. Stromal tissue immediately adjacent to tumor tissue was also analyzed on the same slide. One of the authors (J.D.M.) reviewed all of the slides in a blinded manner. Immunohistochemical detection of COX-2 was performed by using the tyramide signal amplification indirect amplification method. ${ }^{25}$ The sections were deparaffinized and rehydrated and sequentially treated to block endogenous peroxidase (3\% hydrogen peroxide in methanol) and biotin (avidin-biotin blocking kit, no. SP-2001, Vector Corp). The sections were then incubated with a monoclonal antibody for COX-2 (Caymen Chemical) at $4{ }^{\circ} \mathrm{C}$ overnight, followed by incubation with a labeled polymer, horseradish peroxidase (anti-mouse) (DAKO Corp), for 1 hour. Tissue sections were then treated in strepavidin-peroxidase for 30 minutes at room temperature, incubated with biotinylated tyramide at a 1:50 dilution for 15 minutes at room temperature, and finally incubated in strepavidin-peroxidase for 30 minutes at a dilution of 1:250. After 


\begin{tabular}{|c|c|c|c|}
\hline & \multicolumn{3}{|c|}{ Squamous cell } \\
\hline & $\begin{array}{l}\text { Adenocarcinoma } \\
\qquad(\mathrm{n}=28)\end{array}$ & $\begin{array}{c}\text { carcinoma } \\
(\mathrm{n}=18)\end{array}$ & $\begin{array}{c}\text { Total } \\
(n=46)\end{array}$ \\
\hline $\begin{array}{l}\text { Mean age (y) } \\
\text { Male/female }\end{array}$ & $\begin{array}{c}63 \text { (range, 39-80) } \\
24: 4\end{array}$ & $\begin{array}{c}62 \text { (range, 51-78) } \\
11: 7\end{array}$ & $\begin{array}{c}62 \text { (range, 39-80) } \\
35: 11\end{array}$ \\
\hline Median follow-up (mo) & 18 (range, 1-77) & 13 (range, 1-35) & 16 (range, 1-77) \\
\hline \multicolumn{4}{|l|}{ Response to neoadjuvant therapy } \\
\hline Complete pathologic response & $9(32 \%)$ & $3(17 \%)$ & $12(26 \%)$ \\
\hline Partial pathologic response (microscopic residual tumor) & $6(21 \%)$ & $7(39 \%)$ & $13(28 \%)$ \\
\hline No response (gross residual tumor) & $13(46 \%)$ & $8(44 \%)$ & $21(46 \%)$ \\
\hline \multicolumn{4}{|l|}{ Clinical status } \\
\hline Alive without disease & $15(54 \%)$ & $10(56 \%)$ & $25(54 \%)$ \\
\hline Alive with disease & 0 & $1(6 \%)$ & $1(2 \%)$ \\
\hline Dead of disease & $12(43 \%)$ & $6(33 \%)$ & $18(39 \%)$ \\
\hline Dead of other causes & $1(4 \%)$ & $1(6 \%)$ & $2(4 \%)$ \\
\hline
\end{tabular}

staining with $\mathrm{DAB}$, followed by a light hematoxylin counterstain, the sections were dehydrated and placed in coverslips. A case of colon carcinoma known to strongly express COX-2 was used as a positive control. Omission of the primary antibody was used as the negative control. The cases were scored according to the proportion of either tumor or stromal cells that stained for COX-2 $(0$, negative; $1, \leq 33 \% ; 2,34 \%-66 \%$; and $3, \geq 67 \%$ ).

Immunohistochemistry for VEGF was carried out by using a rabbit polyclonal antibody for VEGF (VEGF A-20, Santa Cruz Antibodies) at a 1:100 dilution after antigen retrieval by means of the microwave technique $(750 \mathrm{~W}$ for 5 minutes in $\mathrm{pH} 6.0$ citrate buffer). The secondary antibody and development were combined in the DAKO EnVision+ system (DAKO Corp). A light hematoxylin stain was used as the counterstain. A case of colon carcinoma known to strongly express VEGF was used as a positive control. Omission of the primary antibody was used as a negative control. The scoring of VEGF expression was based on the German Reactive Scoring System, in which the intensity of staining was graded on a scale of 0 to 3 (0, no staining; 1, weak; 2, moderate; and 3, strong), and the percentage of stained cells was graded on a scale of 0 to $4(0,0 ; 1,1 \%-10 \% ; 2,11 \%-50 \% ; 3$, $51 \%-80 \%$; and $4,81 \%-100 \%) .{ }^{26}$ The intensity and percentage of cells that stained positive were multiplied to arrive at a final score that ranged from 0 to 12 .

The microvessel density of the tumors and stroma was evaluated by examining CD31 expression. A monoclonal antibody for CD31 (Neomarkers, Inc) was incubated with the tissue sections at $4^{\circ} \mathrm{C}$ overnight at a 1:500 dilution, followed by incubation with a biotinylated anti-mouse antibody for 1 hour at a 1:100 dilution. The reaction was developed with an avidin-biotin development system, followed by a light hematoxylin counterstaining. Microvessel density was calculated according to previously published procedures. ${ }^{27}$ The areas of tumor with the most extensive neovascularization, termed hot spots, were identified by scanning stained slides at low power. These areas were generally found at the periphery, or the deepest invasive margin, of the tumor. In these hot spot areas, the number of microvessels was counted per $40 \times$ microsopic field. At least 3 different $40 \times$ fields, or hot spots, were evaluated for each case to obtain the mean microvessel count for each patient.

\section{Statistical Methods}

Data analyses were performed with STATA software (Stata Press). Matched comparisons between pretreatment and posttreatment expression and between expression in tumor and stroma or inflammatory tissue were tested with the signed-rank test. Nonmatched comparisons were tested with the Mann-Whitney test. The prognostic significance of various expression levels was tested with the Cox regression model.

\section{Results}

\section{Clinical and Pathologic Features of the Study Population}

Of the 46 patients in the study, 28 had adenocarcinoma, and 18 had squamous cell carcinoma (Table 1). The mean age was 62 years, and there was a clear male preponderance (male/female ratio, 35:11). The median follow-up was 18 months (range, 1-77 months) for the 28 patients with adenocarcinoma and 13 months (range, 1-35 months) for the 18 patients with squamous cell carcinoma. All patients were confirmed through a medical record review to have received preoperative concurrent chemotherapy and radiation. Twelve $(26 \%)$ patients had complete pathologic responses to neoadjuvant therapy, with no residual tumor cells present in the resection specimen. Thirteen $(29 \%)$ patients had a partial response to therapy (microscopic residual tumor), and $21(46 \%)$ patients had minimal or no response to therapy (gross residual tumor). Because several patients had received their preoperative chemotherapy outside our institution, the specific preoperative chemotherapy regimens were obtainable in only $31(67 \%)$ patients. As anticipated, the majority (28 [90\%] patients) received treatment with the standard regimen of cisplatin, 5-fluorouracil, and external beam radiation. The remaining $3(10 \%)$ patients received 5 -fluorouracil alone in combination with external beam radiation. 
TABLE 2. Changes in VEGF and COX-2 expression before and after preoperative chemoradiation

\begin{tabular}{|c|c|c|c|c|c|c|}
\hline \multirow[b]{2}{*}{ VEGF expression } & \multicolumn{3}{|c|}{ Before treatment } & \multicolumn{3}{|c|}{ After treatment } \\
\hline & $\mathbf{N}$ & $\begin{array}{c}\text { Cases expressing } \\
\text { VEGF }(\%)\end{array}$ & $\begin{array}{c}\text { Mean VEGF } \\
\text { expression score }\end{array}$ & $\mathbf{N}$ & $\begin{array}{c}\text { Cases expressing } \\
\text { VEGF }(\%)\end{array}$ & $\begin{array}{c}\text { Mean VEGF } \\
\text { expression score }\end{array}$ \\
\hline \multicolumn{7}{|l|}{ Tumor } \\
\hline Adenocarcinoma & 28 & $18(64)$ & 2.8 & 12 & $11(92)$ & $8.3(P=.007)$ \\
\hline SCCa & 18 & $10(56)$ & 3.3 & 8 & $8(100)$ & $7.3(P=.01)$ \\
\hline Total & 46 & $28(61)$ & 3.0 & 20 & $19(95)$ & $7.9(P=.0002)$ \\
\hline \multicolumn{7}{|c|}{ Tumor-associated stroma } \\
\hline Adenocarcinoma & 28 & $16(57)$ & 0.82 & 28 & $28(100)$ & $3.3(P=.0001)$ \\
\hline $\mathrm{SCCa}$ & 18 & $11(61)$ & 0.67 & 18 & $18(100)$ & $4.4(P=.0002)$ \\
\hline Total & 46 & $27(59)$ & 0.76 & 46 & $46(100)$ & $3.7(P<.0001)$ \\
\hline & & Cases expressing & Mean COX-2 & & Cases expressing & Mean COX-2 \\
\hline COX-2 expression & $\mathbf{N}$ & COX-2 (\%) & expression score & $\mathbf{N}$ & COX-2 (\%) & expression score \\
\hline \multicolumn{7}{|l|}{ Tumor } \\
\hline Adenocarcinoma & 28 & $11(39)$ & 0.41 & 16 & $10(63)$ & $0.78(P=.19)$ \\
\hline $\mathrm{SCCa}$ & 18 & $13(72)$ & 1.0 & 9 & $6(67)$ & $1.0(P=.95)$ \\
\hline Total & 46 & $24(52)$ & 0.65 & 25 & $16(64)$ & $0.86(P=.28)$ \\
\hline \multicolumn{7}{|c|}{ Tumor-associated stroma } \\
\hline Adenocarcinoma & 28 & $10(36)$ & 0.52 & 28 & $22(79)$ & $1.3(P=.003)$ \\
\hline SCCa & 18 & $0(0)$ & 0 & 18 & $16(89)$ & $1.4(P=.0002)$ \\
\hline Total & 46 & $10(22)$ & 0.32 & 46 & $38(83)$ & $1.4(P<.0001)$ \\
\hline
\end{tabular}

VEGF, Vascular endothelial growth factor; COX-2, Cyclooxygenase 2; SCCa, Squamous cell carcinoma.

\section{VEGF Expression}

Tumor VEGF expression was evaluated in 46 pretreatment biopsy specimens. The proportion of tumors expressing VEGF significantly increased after preoperative treatment: before treatment, $28(61 \%)$ of 46 biopsy specimens expressed VEGF; after treatment, 19 (95\%) of 20 specimens expressed VEGF (Table 2). The mean level of VEGF expression, as measured by the mean VEGF expression score, also increased after treatment, from a pretreatment level of 3.0 to a posttreatment level of 7.9. $(P=.0002)$.

In 26 cases posttreatment tumor specimens could not be evaluated for VEGF staining because of treatment effect, resulting in either insufficient tumor cells for accurate analysis or, in the case of complete responders, absent residual tumor cells. We therefore separately evaluated the VEGF staining scores in the 20 patients who had tumors that could be evaluated both before and after treatment. VEGF expression was present in $12(60 \%)$ of 20 of these patients, a proportion that did not significantly differ from the overall cohort. The distribution of VEGF staining scores in these 20 patients illustrates the increase in both the number of positively staining tumors and in the intensity of VEGF expression after preoperative therapy (Figure 1, A).

Preoperative treatment also induced VEGF expression in tumor-associated stromal cells (Table 2). Stromal cells could be evaluated for VEGF expression in 46 cases both before and after treatment. Preoperative therapy resulted in an increase in the proportion of stromal cells expressing VEGF: before treatment, stromal VEGF expression was observed in 27 (59\%) of 46 cases; after therapy, 46 (100\%) of 46 cases expressed VEGF. The mean VEGF expression score increased from a pretreatment level of 0.76 to a posttreatment level of $3.7(P<.0001)$. The distribution of stromal VEGF expression scores illustrates the increase in both the number of tumors expressing VEGF and in the VEGF staining intensity after preoperative treatment (Figure $1, B)$. Representative VEGF staining results for both tumor and stroma are shown in Figure 2, A.

\section{COX-2 Expression}

Tumor COX-2 expression could be evaluated in 46 pretreatment biopsy specimens and in 25 posttreatment resection specimens (Table 2). No significant induction of tumor COX-2 expresssion was observed. COX-2 expression was noted in $24(52 \%)$ of 46 tumors before treatment compared with $16(64 \%)$ of 25 tumors after treatment. The mean COX-2 expression score before therapy was 0.65 compared with 0.86 after therapy, a difference that did not reach statistical significance.

In 21 cases posttreatment COX-2 levels could not be evaluated after treatment because of insufficient or absent residual tumor cells. Of the 25 cases that could be evaluated for expression both before and after treatment, 10 (40\%) overexpressed COX-2, a proportion that did not differ significantly from the overall cohort. When the distribution of COX-2 expression scores was analyzed in these 25 tumors before and after treatment, no clear difference in overall staining intensity was noted (Figure 1,C). 
A.) VEGF: Tumor $(n=20)$

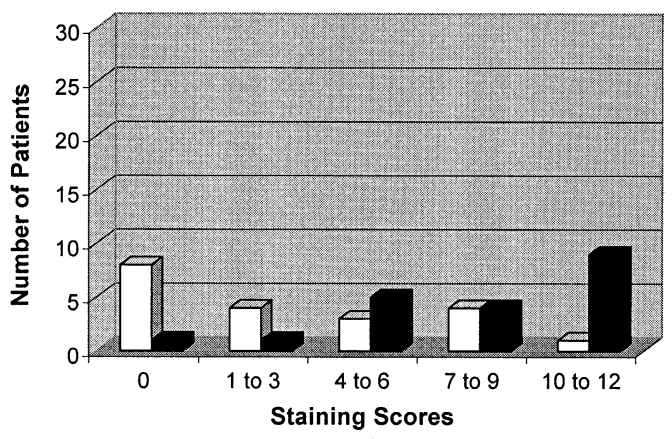

C.) CoX-2: Tumor $(n=25)$

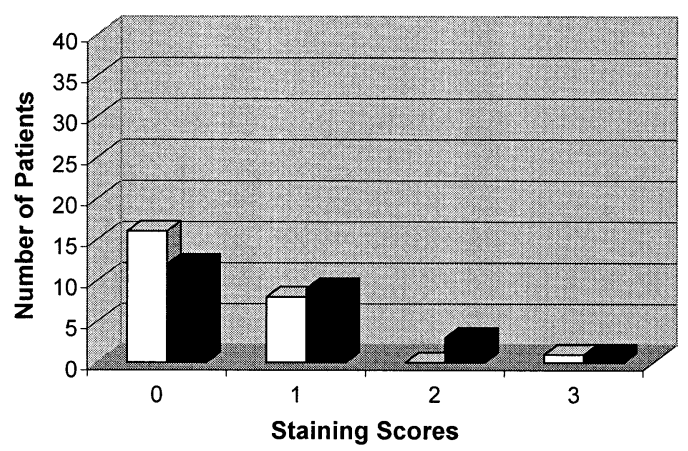

B.) VEGF Stroma $(n=46)$

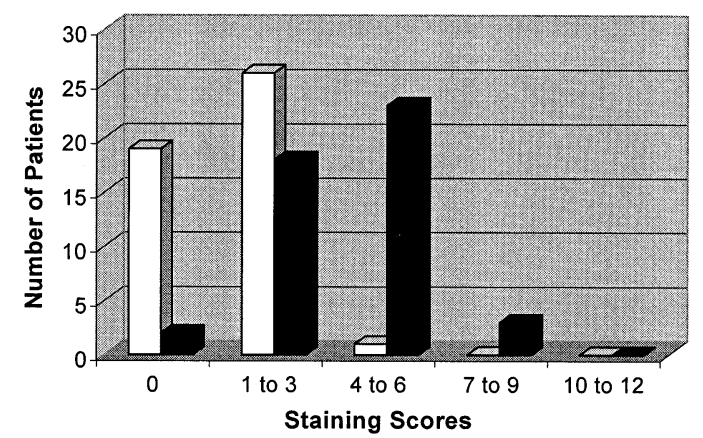

D.) COX-2: Stroma $(n=46)$

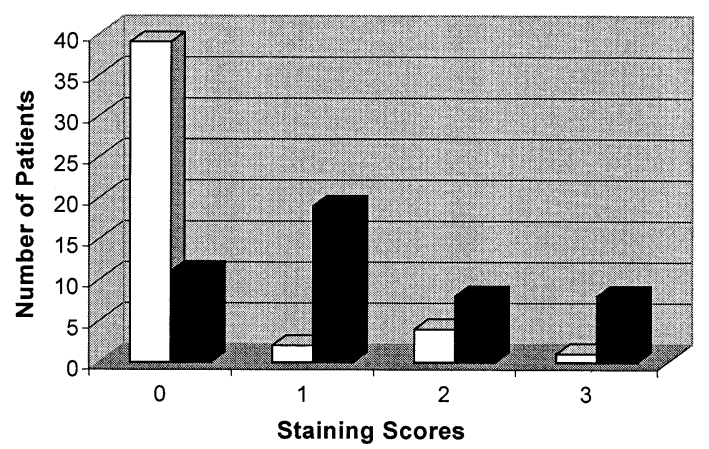

Figure 1. Distribution of staining scores in cases that could be evaluated both before and after preoperative chemotherapy and radiation: A, VEGF tumor $(n=20)$; B, VEGF stroma $(n=46)$; C, COX-2 tumor $(n=25)$; D, COX-2 stroma ( $=46$ ). White bars, pretreatment; black bars, posttreatment.

Stromal COX-2 expression could be evaluated in the pretreatment biopsy specimens and posttreatment resection specimens of all 46 cases (Table 2). Preoperative therapy resulted in significant induction of stromal COX-2 expression. Before treatment, $10(36 \%)$ of 46 cases expressed COX-2 compared with $38(83 \%)$ of 46 cases after treatment. The mean stromal COX-2 expression score increased from a pretreatment level of 0.32 to a posttreatment level of 1.4 $(P<.0001)$. The distribution of stromal COX-2 expression scores further illustrates the increase in both the proportion and intensity of stromal COX-2 staining after treatment (Figure 1,D). Representative COX-2 staining results for both tumor and stroma are shown in Figure 2, B.

\section{Stromal Microvessel Density}

We anticipated that increased stromal VEGF and COX-2 expression would correlate closely with increased angiogenesis and increased microvessel density in stromal tissue. Measurements of stromal CD31 expression in our samples confirmed that stromal microvessel density increased in parallel with VEGF and COX-2 expression: the mean microvessel density score in 38 cases that could be evaluated was 17.2 before treatment compared with 20.6 after preoperative therapy $(P=.02)$.

\section{Prognostic Significance of VEGF and COX-2 Expression}

Low levels of tumor COX-2 before treatment were predictive for treatment response, defined as complete pathologic response, among patients with squamous cell carcinoma (Table 3). Pretreatment levels of tumor COX-2 were not predictive of response among patients with adenocarcinoma. We found no correlation between pretreatment tumor VEGF expression and response to therapy. Patients with high pretreatment levels of VEGF and COX-2 tended to have shorter overall survival times; however, this trend did not reach statistical significance.

\section{Discussion}

In this study we evaluated the effect of preoperative chemoradiation on VEGF and COX-2 expression in patients with esophageal cancer and assessed whether pretreatment expression levels correlated with treatment response or overall survival. We found no association between VEGF expression and treatment response. Although absence of COX-2 expression was associated with complete pathologic response in the subset of patients with squamous cell carcinoma, there was no correlation between COX-2 expres- 


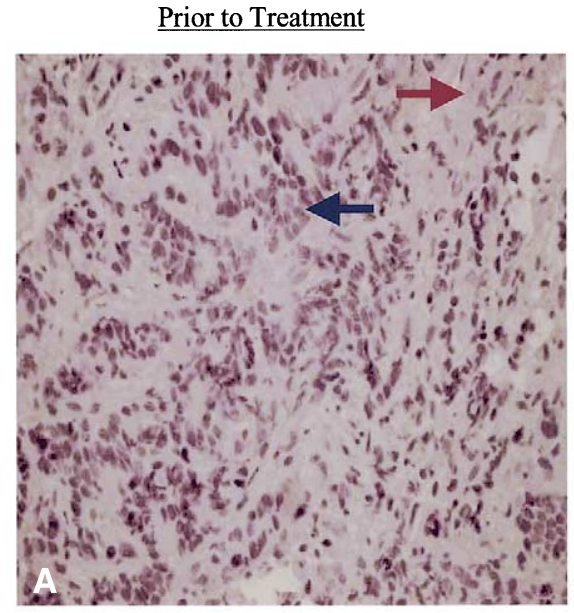

Prior to Treatment

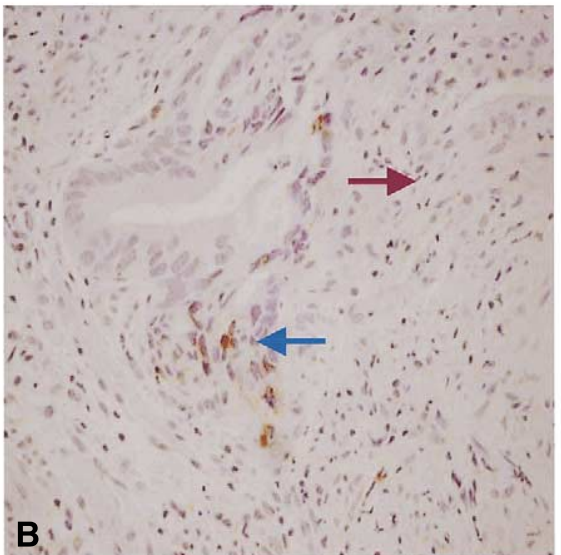

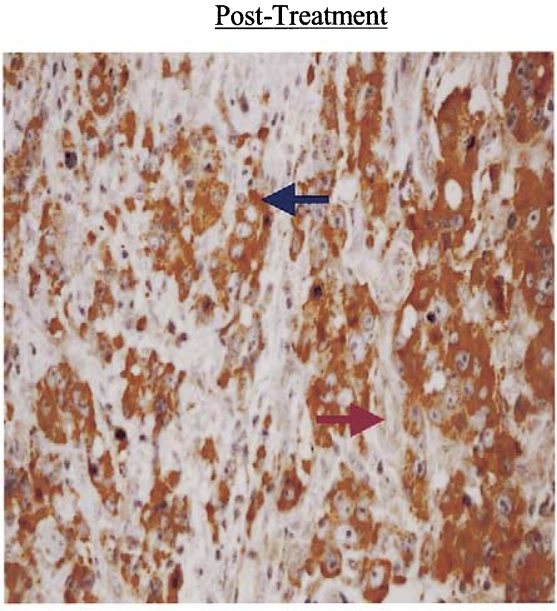

Post Treatment

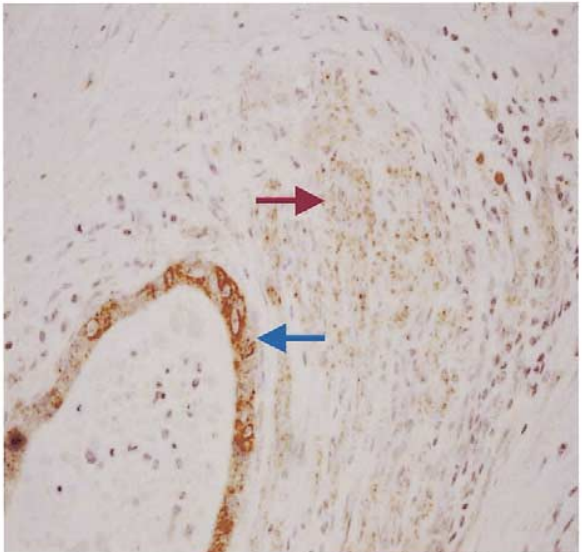

Figure 2. Expression of VEGF and COX-2 before and after preoperative chemoradiation. A, Immunohistochemical staining with antibodies to VEGF. A preoperative biopsy specimen demonstrates no staining of either epithelial cells (blue arrow) or stroma (red arrow). A posttreatment resection specimen of the same case demonstrates strong staining in epithelial cells (blue arrow), as well as staining of stroma (red arrow). (Original magnification $400 \times$ for both photos.) B, Immunohistochemical staining with antibodies to COX-2. A preoperative biopsy specimen demonstrates scattered positive epithelial cells (blue arrow) and no staining in stroma (red arrow). A posttreatment resection specimen of the same case demonstrates staining in epithelial cells (blue arrow), as well as stroma (red arrow). (Original magnification $400 \times$ for both photos.)

sion and response in patients with adenocarcinoma. Patients whose tumors expressed COX-2 and VEGF before treatment tended to have shorter median survival times, but these differences did not reach statistical significance.

Most previous studies have found that VEGF overexpression is an adverse prognostic factor in patients with esophageal cancer. This association is presumably due to the fact that increased tumor angiogenesis reflects more aggressive clinical behavior. ${ }^{6-11}$ At least 2 small studies of preoperative chemoradiation in patients with esophageal cancer have suggested an association between high VEGF levels and poor prognosis. ${ }^{11,12}$ Interestingly, a larger Japanese study of 73 patients with esophageal cancer receiving preoperative chemoradiation failed to find such an association. ${ }^{13}$ Similarly, although we noted a tendency for patients with VEGF overexpression to have shorter median survival times, this association did not reach statistical significance.

The lack of a clear correlation between pretreatment VEGF levels and either treatment response or survival in these studies might, in part, be explained by our observation that preoperative chemotherapy and radiation induce expression of VEGF. Such induction results in increased angiogeneic activity, as demonstrated by a parallel increase in microvessel density. Similar findings have been reported in studies of human xenografts, which have demonstrated that treatment, particularly radiation, induces VEGF expression, 
TABLE 3. Association between pretreatment expression levels of COX-2 and VEGF, treatment response, and survival in patients with esophageal cancer

\begin{tabular}{|c|c|c|c|c|c|c|c|}
\hline \multirow[b]{2}{*}{ Tumor type } & \multirow[b]{2}{*}{$\mathbf{N}$} & \multicolumn{3}{|c|}{$\begin{array}{c}\text { Number }(\%) \text { with complete pathologic response to } \\
\text { preoperative therapy as a function of marker } \\
\text { expression }\end{array}$} & \multicolumn{3}{|c|}{$\begin{array}{c}\text { Median-survival duration as a function of marker } \\
\text { expression }\end{array}$} \\
\hline & & COX-2 positive & COX-2 negative & $P$ value & COX-2 positive & COX-2 negative & $P$ value \\
\hline Adenocarcinoma & 28 & $4 / 11(36)$ & $5 / 17(29)$ & 1.00 & $10 \mathrm{mo}$ & $14 \mathrm{mo}$ & .28 \\
\hline $\begin{array}{l}\text { Squamous cell } \\
\text { carcinoma }\end{array}$ & 18 & $0 / 13(0)$ & $3 / 5(60)$ & .01 & $8 \mathrm{mo}$ & $11 \mathrm{mo}$ & .24 \\
\hline \multirow[t]{2}{*}{ Total } & 46 & $4 / 24(17)$ & $8 / 22(36)$ & .18 & $8 \mathrm{mo}$ & $13.5 \mathrm{mo}$ & .14 \\
\hline & & VEGF positive & VEGF negative & $P$ value & VEGF positive & VEGF negative & $P$ value \\
\hline Adenocarcinoma & 28 & 6/18 (33) & $3 / 10(30)$ & 1.00 & $10.5 \mathrm{mo}$ & $12 \mathrm{mo}$ & .90 \\
\hline $\begin{array}{l}\text { Squamous cell } \\
\text { carcinoma }\end{array}$ & 18 & $3 / 10(30)$ & $0 / 8(0)$ & .22 & $8.5 \mathrm{mo}$ & $13.5 \mathrm{mo}$ & .27 \\
\hline Total & 46 & $9 / 28(32)$ & $3 / 18(17)$ & .32 & $9 \mathrm{mo}$ & $12 \mathrm{mo}$ & .39 \\
\hline
\end{tabular}

$P$ value for response rate was determined by using the Fisher's exact test. $P$ value for comparison of survival duration was determined by using the Mann-Whitney test. COX-2, Cyclooxygenase 2; VEGF, vascular endothelial growth factor.

increases angiogenesis, and potentiates the growth of treatment-resistant tumor cells. ${ }^{28,29}$ These models suggest that the treatment-induced development of more aggressive and resistant tumor phenotypes might weaken potential associations between pretreatment VEGF levels, treatment response, and overall survival.

Unlike VEGF expression, which increased in both tumor and stromal tissue after preoperative treatment with chemotherapy and radiation, COX-2 expression increased in tumor-associated stroma and inflammatory cells but remained relatively stable in the tumor tissue that remained after therapy. We found that low COX-2 expression was associated with complete pathologic response in the subset of patients with squamous cell carcinoma of the esophagus. This observation is limited by the relatively small number of patients who could be evaluated in this cohort but is consistent with recent findings in patients undergoing treatment for squamous cell carcinoma of the cervix, in which COX-2 overexpression was also associated with resistance to chemoradiation. ${ }^{20}$ We found no association between COX-2 expression and treatment response in patients with adenocarcinoma. As with VEGF, the tendency for patients with COX-2 overexpression to have shorter median survival times did not reach statistical significance. Whether the induction of COX-2 in tumor-associated stromal tissue might also lead to the development of treatment resistance and weaken a potential association between pretreatment tumor levels of COX-2 and survival is uncertain.

In summary, we found that VEGF expression is not predictive of treatment response and does not strongly correlate with survival in patients undergoing preoperative chemoradiation for esophageal cancer. Low levels of COX-2 expression do appear to correlate with treatment response in the subset of patients with squamous cell car- cinoma but, like VEGF, do not strongly correlate with survival. Changes in the expression levels of VEGF and COX -2 induced by preoperative therapy might explain their lack of strong prognostic value.

\section{References}

1. Jemal A, Thomas A, Murray T, Thun M. Cancer statistics, 2002. $C A$ Cancer J Clin. 2002;52:23-47.

2. Heath E, Burtness B, Heitmiller R, et al. Phase II evaluation of preoperative chemoradiation and postoperative adjuvant therapy for squamous cell and adenocarcinoma of the esophagus. J Clin Oncol. 2000;18:868-76

3. Forastiere A, Heitmiller R, Lee D, et al. Intensive chemoradiation followed by esophagectomy for squamous cell and adenocarcinoma of the esophagus. Cancer J Sci Am. 1997;3:144-52.

4. Leung D, Cachianes G, Kuang W, Goeddel D, Ferrara N. Vascular endothelial growth factor is a secreted angiogenic mitogen. Science. 1989:246:1306-9.

5. Thomas K. Vascular endothelial growth factor, a potent and selective angiogenic agent. J Biol Chem. 1996;271:603-6.

6. Shih C, Ozawa S, Ando N, Ueda M, Kitajima M. Vascular endothelial growth factor expression predicts outcome and lymph node metastasis in squamous cell carcinoma of the esophagus. Clin Cancer Res. 2000;6:1161-8.

7. Kitadai Y, Haruma K, Tokutomi T, et al. Significance of vessel count and vascular endothelial growth factor in human esophageal carcinomas. Clin Cancer Res. 1998;4:2195-200.

8. Inoue K, Ozeki Y, Suganuma T, Sugiura Y, Tanaka S. Vascular endothelial growth factor expression in primary esophageal squamous cell carcinoma. Cancer. 1997;79:206-13.

9. Uchida S, Shimada Y, Watanabe G, et al. In oesophageal squamous cell carcinoma vascular endothelial growth factor is associated with p53 mutation, advanced stage, and poor prognosis. $\mathrm{Br} J$ Cancer. 1998;77:1704-9.

10. Sato F, Shimada $Y$, Watanabe G, Uchida S, Makino T, Imamura M. Expression of vascular endothelial growth factor, matrix metalloproteinase-9, and E-cadherin in the process of lymph node metastasis in oesophageal cancer. Br J Cancer. 1999;80:1366-72.

11. Shimada H, Hoshino T, Okazumi S, et al. Expression of angiogenic factors predicts response to chemoradiotherapy and prognosis of oesophageal squamous cell carcinoma. Br J Cancer. 2002;86:552-7.

12. Imdahl A, Bognar G, Schulte-Montig J, Schoffel U, Farthmann E, 
Ihling C. Predictive factors for response to neoadjuvant therapy in patients with oesophageal cancer. Eur J Cardiothorac Surg. 2002;21: 657-63.

13. Hironaka S, Hasabe T, Kamijo T, et al. Biopsy specimen microvessel density is a useful prognostic marker in patients with T2-4M0 esophageal cancer treated with chemoradiotherapy. Clin Cancer Res. 2002; 8:124-30.

14. Torres C, Wang H, Turner J, Shahsafaei A, Odze R. Prognostic significance and effect of chemoradiotherapy on microvessel density (angiogenesis) in esophageal Barrett's esophagus-associated adenocarcinoma and squamous cell carcinoma. Hum Pathol. 1999;30:753-8.

15. Masferrer J, Leahy K, Koki A, et al. Antiangiogenic and antitumor activities of cyclooxygenase-2 inhibitors. Cancer Res. 2000;60:130611.

16. Leahy K, Ornberg R, Wang Y, Zweifel B, Koki A, Masferrer J. Cyclooxygenase-2 inhibition by celecoxib reduces proliferation and induces apoptosis in angiogenic endothelial cells in vivo. Cancer Res. 2002;62:625-31.

17. Shi $\mathrm{H}, \mathrm{Xu} \mathrm{J}, \mathrm{Hu} \mathrm{N}$, Xie H. Prognostic significance of expression of cyclooxygenase-2 and vascular endothelial growth factor in human gastric carcinoma. World J Gastroenterol. 2003;9:1421-6.

18. Denkert C, Winzer K, Muller B, et al. Elevated expression of cyclooxygenase-2 is a negative prognostic factor for disease free survival and overall survival in patients with breast carcinoma. Cancer. 2003; 97:2978-87.

19. Kim S, Kwon S, Kim Y, Hong S. Association of cyclooxygenase-2 expression with prognosis of stage T1 grade 3 bladder cancer. Urology. 2002;60:816-21.

20. Ferrandia G, Lauriola L, Distefano M, et al. Increased cyclooxygenase-2 expression is associated with chemotherapy resistance and poor survival in cervical cancer patients. J Clin Oncol. 2002;20:973-81.

21. Gallo O, Masini E, Bianchi B, Bruschini L, Paglierani M, Franchi A.
Prognostic significance of cyclooxygenase-2 pathway and angiogenesis in head and neck squamous cell carcinoma. Hum Pathol. 2002;33: 708-14.

22. Ferrandina G, Laurioloa L, Zannoni G, et al. Increased cyclooxygenase-2 (COX-2) expression is associated with chemotherapy resistance and outcome in ovarian cancer patients. Ann Oncol. 2002;13:1205-11.

23. Kim Y, Kim G, Cho N, et al. Overexpression of cyclooxygenase-2 is associated with a poor prognosis in patients with squamous cell carcinoma of the uterine cervix treated with radiation and concurrent chemotherapy. Cancer. 2002;95:531-9.

24. Buskens C, VanRees B, Sivula A, et al. Prognostic significance of elevated cyclooxygenase 2 expression in patients with adenocarcinoma of the esophagus. Gastroenterology. 2002;122:1800-7.

25. Khan K, Masferrer J, Woerner B, Soslow R, Koki A. Enhanced cyclooxygenase-2 expression in sporadic and familial adenomatous polyposis of the human colon. Scand J Gastroenterol. 2001;36:865-9.

26. Remmele W, Stegner H. Vorschlag zur einheitlichen Definition eines Immunreativen Score (IRS) fur den immunohistochemischen Ostrogenrezeptor-Nachweis (ER-ICA) im Mammakarzinomgewebe. Pathologe. 1987;8:138-40.

27. Gelb A, Sudilovsky D, Wu C, Weiss L, Medeiros L. Appraisal of intratumoral microvessel density, MIB-1 score, DNA content, and p53 protein expression as prognostic indicators in patients with locally confined renal cell carcinoma. Cancer. 1997;80:1768-75.

28. Griffin R, Williams B, Wild R, Cherrington J, Park H, Song C. Simultaneous inhibition of the receptor kinase activity of vascular endothelial, fibroblast, and platelet-derived growth factors suppresses tumor growth and enhances radiation response. Cancer Res. 2002;62: 1702-6.

29. Gorski D, Beckett M, Jaskowiak N, et al. Blockade of the vascular endothelial growth factor stress response increases the antitumor effects of ionizing radiation. Cancer Res. 1999;59:3374-8. 